\title{
DISPLAYING DIII-D PLASMA DATA USING DEC's $X$ WINDOW SYSTEM
}

\author{
by \\ K.L. GREENE
}

This is a preprint of a paper to be presented at the 14th Symposium on Fusion Engineering, September 30-October 3, 1991, San Diego, California, and to be printed in the Proceedings.

\author{
Work supported by \\ Department of Energy \\ Contract DE-AC03-89ER51114
}
GENERAL ATOMICS PROJECT 3466 NOVEMBER 1991

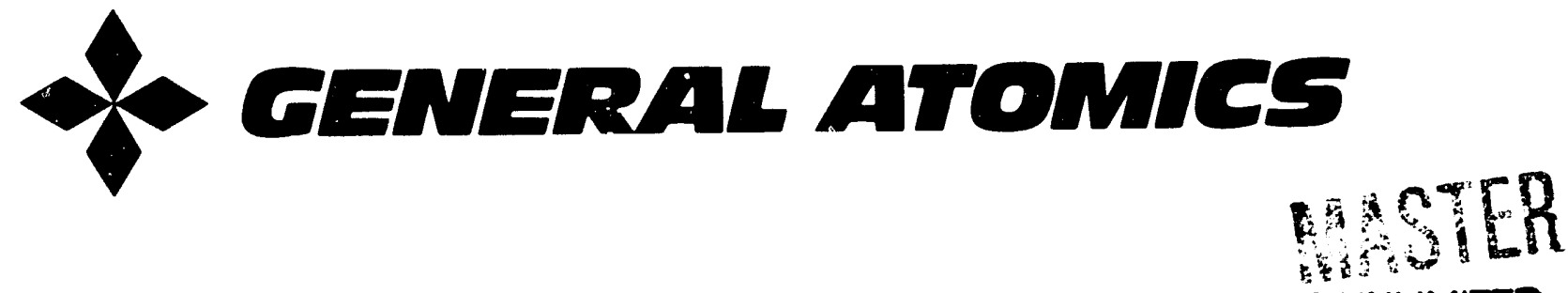




\title{
DISPLAYING DIII-D PLASMA DATA USING DEC's $X$ WINDOW SYSTEM
}

\author{
Katherine L. Greene \\ General Atomics \\ P.O. Box 85608 \\ San Diego, California 92186-9784
}

Abstract: The DIII-D tokamak program, funded by the Department of Energy, carries out plasma physics and fusion energy research experiments. The machine began operation in February 1986; at that time, approximately 7 Mbytes of data was collected for each shot. Since that time, the shot size has steadily increased to over 50 Mbytes with the average shot size between 35 and 45 Mbytes. Shots are fired every 12 to $15 \mathrm{~min}$ utes and last approximately 5 to 10 seconds. Between 30 and 40 shots aie fired each day when plasma experiments are scheduled.

In 1987, MFITD and MFITPLAY were written/modified. These two programs provide graphical output that allows the users to see, before the next shot, the plasma shape and the locations of the plasma and magnetic flux lines within the tolka. mak. MFITD performs the computations which calculate the shape and location of the plasma; it also graphically displeiys a small subset of timeslice data. MFITPLAY graphically displays the full set of timeslice data. Through interactive commands, MFITPLAY also allows the user to control the various aspects of how the data is displayed.

In 1990, both programs were converted from User Interface Services (UIS) routines, which are part of the MicroVMS workstation graphics software, to DEC's X Window System using the DECWindows window manager. These modifications were required because of a move by Digital Equipment Corporation (DEC) to support $X$ windows and phase out UIS. Due to the nature and purpose of each program, MFITD needed only simple graphics conversion while MFITPLAY was completely rewritten. The DECWindows version of MFITPLAY offers a number of improvements, such as a more intuitive user interface.

\section{Introduction}

MFITD and MFITPLAY are programs used by users when running fusion plasma experiments. Both programs display the shape of the tokamak vessel, the shape of the plasma, the location of the plasma within the vessel, the magnetic flux lines, the filaments, the time of the timeslice being displayed, and the shot number. In addition, MFITPLAY displays printed values for 24 of 40 parametrers which are calculated for each timeslice. This information tells the users what happened during the shot and whether they are achieving the desired results of the experiment.

DECWindows came packaged with Version 5.0 of the VMS operating system because DEC planned to move away from UIS and support on , : X Xindows-based Graphical User Interface (GUI). Additionally, only UIS or DECWindows can be installed at any one time. For these reasons, MFITD and MFITPLAY needed to be converted to run under DECWindows.

\section{About the Programs}

MFITD calculates important physics parameters relating to MHD phenomena and makes these results available to the user. The users can control many of the parameters which determine how the calculations are performed. Some of these parameters are start time, delta time, and number of timeslices. Because the users want quick feedback regarding the results of the shot, MFIT J displays some of the data graphically. An additional parameter, delta timeslice, lets the users determine how many timeslices MFITD will display on the workstation's DECWindows terminal.
The results of the calculations performed by MFITD are written to files. The first file contains data in a standard format for data acquisition, i.e., fixed and variable headers followed by data. The headers provide information about the shot such as the date and time when the shot was fired, the type of data (real ${ }^{*} 4$, integer* 2 , etc.), and the total data size. The data acquisition software includes this file in the shot data. The result is that this data can be accessed by many programs, such as general plotting programs or programs written to analyze specific aspects of a shot. The second and third files are used by MFITPLAY. The first of these two files contains the data defining the shape of the tokamak and the locations of the filaments. The second file contains the data for the plasma shape and the flux lines. For each timeslice calculated, the file also contains an array of values fo- 40 parameters giving additional information about the plasma. Figure 1 provides an overview of the relationship between the programs and their input and output.

MFITPLAY displays all the timeslice data calculated by MFITD. For each shot, the program reads the data files written by MFITD and then continuously displays one timeslice after another until it reaches the end of the data. At this point, the program continues to display the same data but now it is displayed in reverse chronological order until the program reaches the beginning of the data. The cycle continues until new shot data is available or until someone halts the program.

At the beginning of a plasma operations day, the computer operator logs in to the workstation and initiates MFITPLAY. The program reads the data for the last shot and begins displaying this data. Every time a timeslice is displayed, MFITPLAY will also check whether the next shot has fired. When it sees a new shot number, it starts looking for the presence of the data files created by MFITD for the new shot. MFITPLAY runs continuously through the day until plasma operations are complete. At that time, the computer operator terminates the program. MFITD, however, is started up as a detached process on a VAXstation 3200 during each shot by the acquisition software, which runs remotely on another VAX. When MFITD has completed all its calculations and has written the various output files, it then terminates.

Originally, both programs had been written using UIS graphical routines. Because DEC planned to phase out UIS and switch to an Xwindows-based GUI, both programs were translated to DECWindows. MFITD required only one window for graphical output and did not require any user interface. Therefore, only a small section of code needed to be modified to delete UIS calls and incorporate DECWindows calls. MFITPLAY, however, displayed more graphical information than MFITD. It also offered interactive control of the display. These features required more extensive use of UIS than was required by MFITD As : result, MFITPLAY was completely rewritten.

\section{Improvements and New Features}

Rewriting MFITPLAY provided the opportunity to modify the basic features of the program, taking into account the preferences expressed by the users when they used the UIS version. Controlling the display of graphics in the UIS version of MFITPLAY often required multiple keystrokes. The user could not type fast enough to maintain accurate control of MFITPLAY's rapid display. For example, if it was desired to stop the 


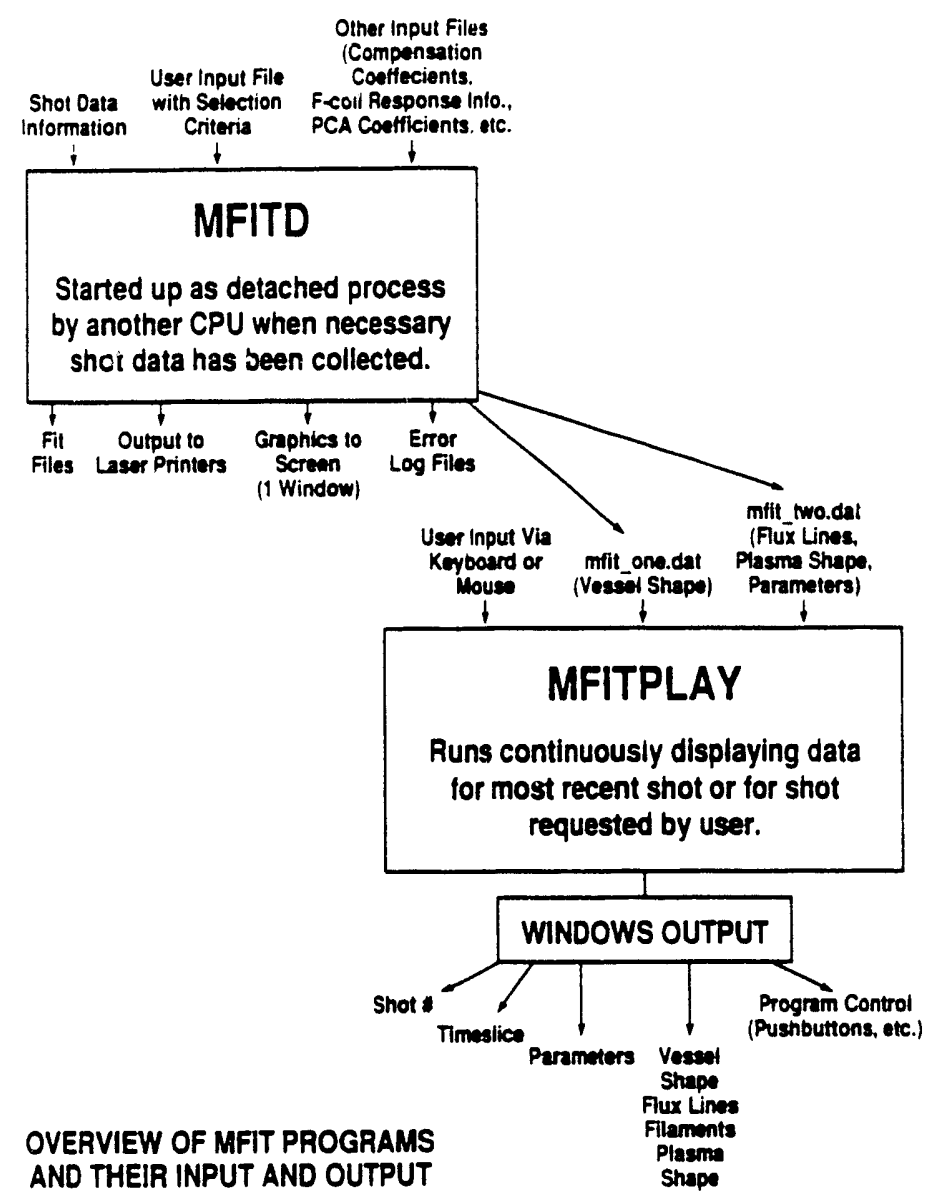

Fig. 1. Overview of MFIT programs and their input and output.

MFITPLAY display on a particular timeslice, by the time the user had entered the proper keystrokes the program had progressed to a timeslice beyond the one desired; and there was no way to move only one timeslice at a time.

The DECWindows version has eliminated these problems. The user can easily position the mouse pointer on the desired program action and click a button. MFITPLAY offers the option of incrementing or decrementing the display by one timeslice through the use of pushbuttons. One of the new features allows the user to type a specific timeslice to be displayed. This is particularly useful if the timeslice of interest is 45 th out of 55 and the program is displaying the third timeslice.

The UIS version allowed changing the shot which was being displayed. The user could increment or decrement the shot number in steps of one. The DECWindows version offers this same option, and in addition, allows the user to request a specific shot number. This new feature is beneficial for two reasons: (1) It saves time. Instead of stepping one shot at a time to go back six shots, the user can go directly to the desired shot. (2) In order to keep the values of internal variables consistent with the information displayed on the screen, neither version allows stepping past a shot for which no data was generated.

The look of the display is also different. All output from the UIS version appeared in a single window. However, the DECWindows version has several windows, each containing related information. The first window contains all the display controls, i.e., push buttons, etc. The second window displays the tokamak vessel, plasma shape, flux lines, and filaments. A third window contains the 24 parameter values. Finally, the shot number and timeslice time are displayed in windows four and five. When MFITD puts its single output window on the screen, it overlays some of MFITPLAY's windows. Putting information in multiple windows allows the user to bring to the foreground of the screen the windows containing the information most desired.

Previously, MFITPLAY would read one record from the data file, display the information, read a record, display the information, etc. Now, the program reads the whole file into memory before displaying the first timeslice. While this causes a small delay before the first timeslice is displayed on the screen, it is more efficient because the file is read only once. This change was also required in order to offer the user the opportunity of requesting a specific timeslice.

\section{Problems and Drawbacks}

Doing any modification to the screen when DECWindows is installed requires a lot of overhead. Since MFITPLAY is continually modifying the screen, approximately $60-80 \%$ of the CPU is taken by DECWSSERVER_0, the process responsible for making all changes to the screen. This greatly impacts the performance of MFITD. Since MFITD is the program which generates the data to be displayed by MFITPLAY, the high overhead required by DECWindows slows the completion of MFITD considerably, thereby delaying when the data can be displayed. For example, with MFITPLAY on hold, i.e., the program remains in memory but does not send any graphical output to the screen, MFITD can calculate data for 49 timeslices in less than three minutes; but when MFITPLAY was allowed to continue normally, MFITD required more than five minutes to complete. Since the users require the information provided by MFITD and MFITPLAY to plan the next shot, these delays can be both irritating and costly. As the total quantity of data collected per shot has increased over the years, the time required to 
acquire the data has also increased, resulting in fewer plasma shots being fired per day. Therefore, the sooner MFITD can complete its calculations, the sooner the users can start to plan the next shot. A solution to this problem is that MFITPLAY will stop all screen output while MFITD is runoing, greatly speeding MFITD's comple: in..

\section{Future Enhancements}

A number of additional features are planned. One of these features is an additional window in which a maximum of 8 of the 40 parameters are plotted vs. time for the whole shot. Another feature, also in its own window, would be a tabular display of all 40 parameters. A third feature would be less visible. Currently, the graphical objects are drawn directly to the window. This results in the time required to display all information for one timeslice to be longer than was required to display the same information under UIS. To speed the output, the third new feature would instead draw the output to a pixmap, an area within memory to be used for creating and modifying images. Then the pixmap would be copied from memory to the window, resulting in faster output.
One of the new features (plotting parameters vs. time) will present a problem in the area of program development. While DECWindows has many graphics routines, there are no predefined routines to draw and label axes and tic marks. These routines will need to be written in-house, delaying the time when this feature can be offered to the users.

\section{Summary}

MFITD and MFITPLAY are now more useful programs. The data is displayed in a more organized form, the interactive controls perform more accurately, the user interface is less complicated, and new features have been added. The ease of use and the additional information help the users to understand what happened in the last shot and to prepare for the next one.

\section{Acknowledgment}

This work was sponsored by the U.S. Department of Energy under Contract No. DE-AC03-89ER51114. 

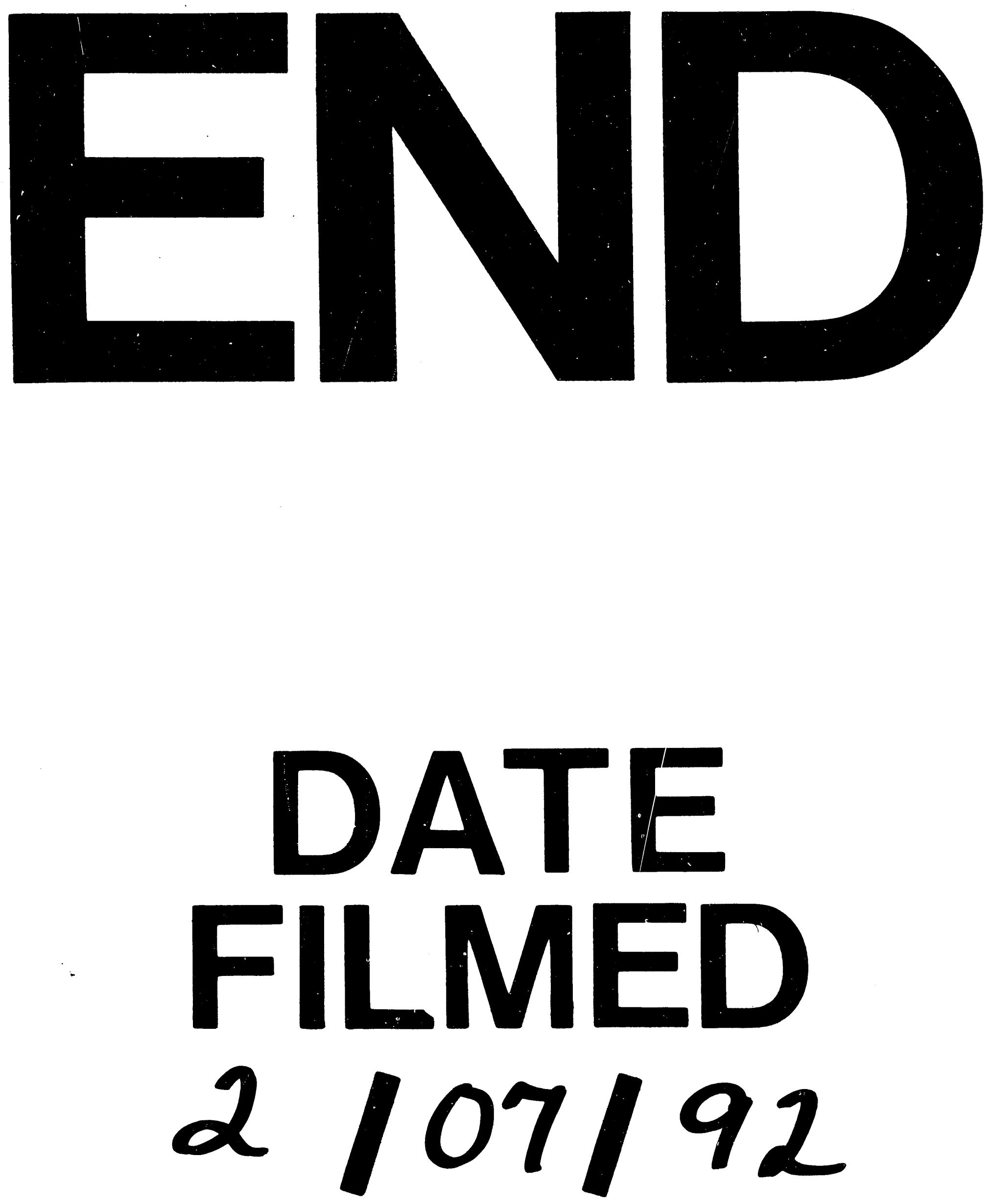

I 
